\title{
Fibroblast activation protein in osteosarcoma cells promotes angiogenesis via AKT and ERK signaling pathways
}

\author{
CHAO ZENG ${ }^{1}$, MING WEN $^{1}$ and XIAOMEI LIU ${ }^{2}$ \\ ${ }^{1}$ Department of Orthopedics, Weifang Traditional Chinese Medical Hospital, Weifang, Shandong 261041; \\ ${ }^{2}$ Oncology and Hematology Department, Huangdao District People's Hospital, Qingdao, Shandong 266000, P.R. China
}

Received April 23, 2016; Accepted January 10, 2018

DOI: $10.3892 / \mathrm{ol} .2018 .8027$

\begin{abstract}
Although it is established as a marker of cancer-associated fibroblasts, the expression of fibroblast activation protein (FAP) is not restricted to stromal cells; its expression in multiple types of tumor cell and its pro-tumor functions have been reported. However, the role of FAP in angiogenesis in osteosarcoma remains uncharacterized. In the present study, it was identified that the mRNA and protein expression levels of FAP and vascular endothelial growth factor-A (VEGF-A) corresponded to each other in MG63, U2-OS and HOS osteosarcoma cells. Subsequent to upregulating FAP in MG63 cells, VEGF-A mRNA and protein expression significantly increased; subsequent to downregulating FAP in U2-OS cells, VEGF-A mRNA and protein expression significantly declined. These changes in VEGF-A level were also detected in the cell supernatant with ELISA. Conditioned medium (CM) from MG63 cells overexpressing FAP promoted the phosphorylation of AKT and extracellular signal-regulated kinase (ERK) in human umbilical vein endothelial cells (HUVECs), as well as the proliferation rate. The CM from U2-OS cells with FAP knockdown inhibited the proliferation rate of HUVECs. The phosphorylation of AKT and ERK was increased in MG63 cells overexpressing FAP, but reduced in U2-OS cells with FAP knockdown. Furthermore, treatment with the AKT inhibitor LY294002 or the ERK inhibitor U0126 inhibited the upregulation of VEGF-A induced by FAP expression. Collectively, the results suggest that FAP expression in osteosarcoma cells promotes angiogenesis.
\end{abstract}

\section{Introduction}

Osteosarcoma, which is derived from primitive bone-forming mesenchymal cells, is the most common type of malignant bone tumor, and predominantly occurs in children and young

Correspondence to: Mr. Chao Zeng, Department of Orthopedics, Weifang Traditional Chinese Medical Hospital, 666 Weizhou Road, Weifang, Shandong 261041, P.R. China

E-mail: zengchaoatweifang@163.com

Key words: fibroblast activation protein, osteosarcoma, angiogenesis, vascular endothelial growth factor-A adults (1). Despite recent developments in medical technology, including surgery and chemotherapy, therapy outcomes remain unsatisfactory; this can be partially attributed to the complex interaction between the tumor cells and the tumor microenvironment (2-4).

Blood vessels are critical component of the tumor microenvironment, as they are not only essential for supplying tumor nutrition, but also for transporting tumor cells to distant sites (5). A growing body of cancer research is focusing on angiogenesis. A range of types of drugs targeting tumor vessels have been developed and applied clinically. Although such drugs improve the treatment outcome to some extent, drug resistance is ultimately induced during treatment, and so therapy outcomes remain unsatisfactory $(6,7)$. Further endeavor is required to identify more effective targets for anti-angiogenesis therapy and improve the prognosis for cancer.

Fibroblast activation protein (FAP) was initially identified as a specific marker for cancer-associated fibroblasts (8). It was previously reported that stromal FAP expression was correlated with the progression and prognosis in a range of tumor types, including colon, breast and ovarian cancer (9-12). In view of the role of stromal FAP in cancer progression, its expression in tumor cells has attracted increasing attention. Mentlein et al (13) reported that FAP was highly expressed on the surface of bone and soft tissue tumor cells. Yuan et al (14) reported that the high expression of FAP in osteosarcoma cells was positively correlated with an advanced clinical stage, high histological grade, positive metastasis and poor prognosis. However, the role of FAP in angiogenesis in osteosarcoma has yet to be characterized.

In the present study, it was identified that FAP expression in osteosarcoma cells promoted the expression of vascular endothelial growth factor-A (VEGF-A). Furthermore, conditioned medium (CM) from osteosarcoma cells with altered FAP expression could activate AKT, ERK and proliferation in endothelial cells. Finally, it was demonstrated that the activation of the AKT and extracellular signal-regulated kinase (ERK) signaling pathways was associated with the VEGF-A expression induced by FAP.

\section{Materials and methods}

Cell lines and cell culture. HOS, U2-OS and MG63 human osteosarcoma cell lines, and human umbilical vein endothelial 
cells (HUVECs) were purchased from American Type Culture Collection (Manassas, VA, USA). HOS, U2-OS and MG63 cells were cultured in Dulbecco's modified Eagle's medium (DMEM; Invitrogen; Thermo Fisher Scientific, Inc., Waltham, MA, USA) supplemented with $10 \%$ fetal bovine serum (FBS; Invitrogen; Thermo Fisher Scientific, Inc.). HUVEC cells were cultured in endothelial cell medium supplemented with $5 \%$ FBS and $1 \%$ endothelial cell growth supplement (all from ScienCell Research Laboratories, Carlsbad, CA, USA). All cells were cultured in $5 \% \mathrm{CO}_{2}$ at $37^{\circ} \mathrm{C}$. To study potential molecular mechanism, the AKT inhibitor LY294002 or ERK inhibitor U0126 were added into osteosarcoma cells using FAP overexpression for $24 \mathrm{~h}$ at $37^{\circ} \mathrm{C}$. The final concentrations of LY294002 and ERK were 50 and $20 \mu \mathrm{M}$, respectively.

Transfection. A total of $2 \times 10^{5}$ cells were plated in 6 -well plates. After $24 \mathrm{~h}, 1.5 \mu \mathrm{g}$ p-cDNA3.1/FAP-vector and $2 \mu \mathrm{l}$ Lipofectamine $^{\circledR} 2000$ (Invitrogen; Thermo Fisher Scientific, Inc.) were mixed for $20 \mathrm{~min}$ at room temperature. Then, the mixture was added in cells in order to overexpress FAP. A total of $1.5 \mu \mathrm{g}$ p-GPU6/FAP-shRNA plasmids (Shanghai GenePharma Co., Ltd., Shanghai, China) and $2 \mu$ l Lipofectamine ${ }^{\circledR} 2000$ were mixed for $20 \mathrm{~min}$ at room temperature. Then, the mixture was added in cells to silence FAP expression. The empty plasmids were used as a control. After $48 \mathrm{~h}$, RNA and protein were extracted for further procedures. Subsequent to culture in DMEM without FBS for a further $48 \mathrm{~h}$, the supernatant was collected as CM. The shRNA sequence is 5'-CCCTCAGAC AGTTTGCTTATT-3'.

Reverse transcription-polymerase chain reaction (RT-PCR). Total RNA from cells was extracted with TRIzol (Invitrogen; Thermo Fisher Scientific, Inc.) according to the manufacturer's protocol.cDNA was synthesized using the PrimeScriptRT-PCR kit (Takara Biotechnology Co., Ltd., Dalian, China) according to the manufacturer's protocol $\left(25^{\circ} \mathrm{C}\right.$ for $10 \mathrm{~min}, 42^{\circ} \mathrm{C}$ for $60 \mathrm{~min}$, then $70^{\circ} \mathrm{C}$ for $10 \mathrm{~min}$ ). The PCR was performed using specific primers and Universal PCR Master Mix (Thermo Fisher Scientific, Inc.) with the following thermocycler conditions: $4^{\circ} \mathrm{C}$ for $5 \mathrm{~min}$, then $94^{\circ} \mathrm{C}$ for $30 \mathrm{sec}, 57^{\circ} \mathrm{C}$ for $30 \mathrm{sec}$ and $72^{\circ} \mathrm{C}$ for $30 \mathrm{sec}$, followed by a holding step at $72^{\circ} \mathrm{C}$ for $5 \mathrm{~min}$. For the FAP PCR, this was repeated for 40 cycles. For the VEGF-A and GAPDH PCR, this was repeated for 36 cycles. PCR products were electrophoretically separated on $1.0 \%$ agarose gels. The results were analyzed by Labwork software, version 4.0 (UVP, Inc., Upland, CA, USA). The primers used were as follows: FAP forwards, 5'-TTAGTCTGACAAAGAGAAACA CTG-3' and reverse, 5'-ATGAAGACTTGGGTAAAAATC G-3'; VEGF-A forwards, 5'-CGGGCAGGAGGAAGGAGC CT-3' and reverse, 5'-GTGATGGTGTGGTGGCGGCA-3'; GAPDH forward, 5'-AGAAGGCTGGGGCTCATTTG-3' and reverse, 5'-AGGGGCCATCCACAGTCTTC-3' GAPDH was used as an internal control.

Western blot analysis. Protein was extracted using radioimmunoprecipitation assay buffer (Beyotime Institute of Biotechnology, Haimen, China) containing 1\% protease inhibitor. Total protein was measured using a bicinchoninic acid assay kit (Pierce; Thermo Fisher Scientific, Inc.) according to the manufacturer's protocol. Then, $300 \mu \mathrm{g} /$ well protein was loaded in 5\% acrylamide and separated by $10 \%$ separating gel, then transferred to a nitrocellulose membrane. Subsequent to blocking in TBS with $0.05 \%$ Tween-20 (ST825, Beyotime Institute of Biotechnology) containing $5 \%$ non-fat dried milk for $1 \mathrm{~h}$ at room temperature, the membrane was then incubated with primary antibodies (listed in Table I) at $4^{\circ} \mathrm{C}$ overnight, followed by incubation with peroxidase-linked goat anti-rabbit-IgG (cat. no. ab205718, dilution, 1:4,000; Abcam, Cambridge, MA, USA) at room temperature for $1 \mathrm{~h}$. Signals were detected using enhanced chemiluminescence reagents (Pierce; Thermo Fisher Scientific, Inc.) and measured using Image-Pro software (version 5.1; Media Cybernetics, Inc., Rockville, MA, USA).

ELISA. VEGF-A in CM was detected using PGRN ELISA kit (cat. no. CSB-E11718h; CUSABIO Life Science, Wuhan, China) and VEGF-A ELISA Kit (CUSABIO Life Science) according to the manufacturer's instructions. The experiment was repeated at least three times.

HUVEC cell proliferation assay. HUVEC cells were plated in a 96-well plate at 5,000 cells per well, in triplicate. Once the cells had adhered to the well walls, the medium was replaced with CM from transfected MG63 or U2-OS cells. An MTT assay was performed at 0 and $48 \mathrm{~h}$ from the addition of CM. Cells were incubated with MTT for $4 \mathrm{~h}$, then formazan was dissolved with $0.1 \%$ dimethyl sulfoxide. The absorbance of the wells was then measured at $490 \mathrm{~nm}$. The proliferation rate was calculated as OD at $48 \mathrm{~h} / \mathrm{OD}$ at $0 \mathrm{~h}$.

Statistical analysis. Data was expressed as the mean \pm standard deviation. SPSS software (version 11.0; SPSS, Inc., Chicago, IL, USA) was used for statistical analysis. Differences between groups were analyzed using one-way analysis of variance with Dunnett's post-hoc test. $\mathrm{P}<0.05$ was considered to indicate a statistically significant difference.

\section{Results}

FAP and VEGF-A expression in osteosarcoma cells. RT-PCR and western blot analysis were performed to detect the expression of FAP and VEGF-A in osteosarcoma cells. As demonstrated in Fig. 1, U2-OS cells exhibited the highest expression of FAP at the mRNA (Fig. 1A and B) and protein (Fig. 1C and D) levels, which was significantly different from MG63 and HOS cells $(\mathrm{P}<0.05)$. MG63 cells exhibited the lowest FAP expression at the mRNA (Fig. 1A and B) and protein (Fig. 1C and D) levels, which were significantly different from U2-OS and HOS cells $(\mathrm{P}<0.05)$. The VEGF-A expression trend among these three cell lines was consistent with FAP expression at the mRNA (Fig. 1A and B) and protein (Fig. 1C and D) levels. This indicated a potential association between FAP and VEGF-A expression in osteosarcoma cells.

FAP promotes VEGF-A expression in osteosarcoma cells. To identify whether an association between FAP and VEGF-A existed, FAP expression was altered by transfection. As demonstrated in Fig. 2, when FAP in MG63 cells 
Table I. Primary antibodies used for western blotting in the present study.

\begin{tabular}{lllll}
\hline Target & \multicolumn{1}{c}{ Specificity } & Dilution & Cat. no. & Company \\
\hline FAP & Rabbit anti-human & $1: 1,000$ & ab53066 & Abcam, Cambridge, MA, USA \\
VEGF-A & Rabbit anti-human & $1: 1,000$ & ab46154 & Abcam \\
Phospho-ERK & Rabbit anti-human & $1: 1,000$ & 4370 & Cell Signaling Technology, Inc., \\
& & & & Danvers, MA, USA \\
ERK & Rabbit anti-human & $1: 1,000$ & 4695 & Cell Signaling Technology, Inc. \\
Phospho-AKT & Rabbit anti-human & $1: 1,000$ & 4058 & Cell Signaling Technology, Inc. \\
AKT & Rabbit anti-human & $1: 1,000$ & 4691 & Cell Signaling Technology, Inc. \\
GAPDH & Rabbit anti-human & $1: 4,000$ & ab128915 & Abcam \\
\hline
\end{tabular}

A

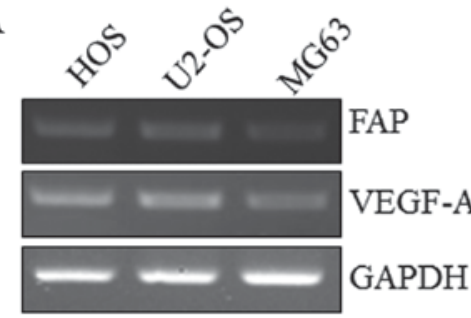

C

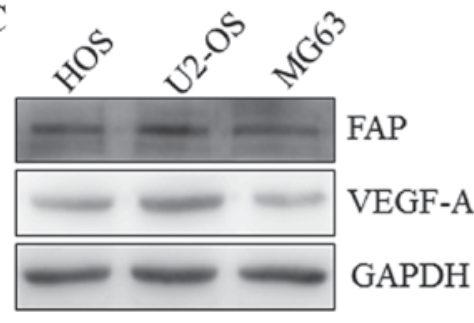

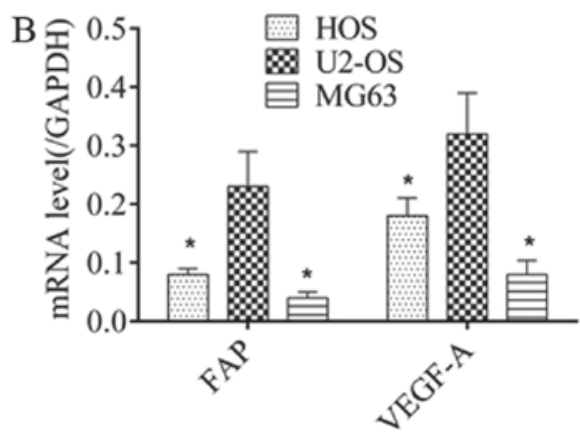

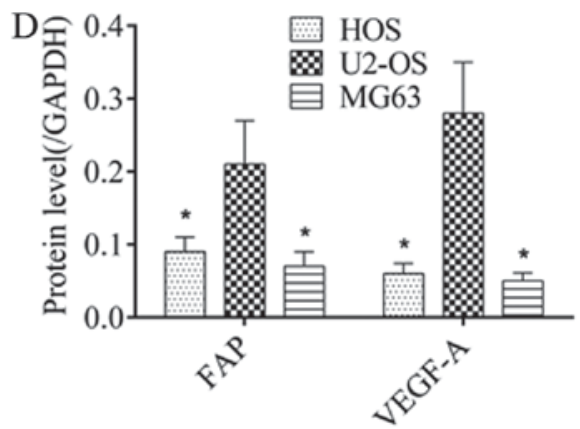

Figure 1. FAP and VEGF-A expression in osteosarcoma cells. FAP and VEGF-A expression were detected in MG63, U2-OS and HOS cell lines. (A) FAP and VEGF-A mRNA expression levels were determined with reverse transcription-polymerase chain reaction, which was (B) quantified by densitometry. (C) FAP and VEGF-A protein expression levels were determined with western blot analysis, which was (D) quantified by densitometry. FAP and VEGF-A in U2-OS cells was compared with the other two groups. ${ }^{*} \mathrm{P}<0.05$ vs. U2-OS. FAP, fibroblast activation protein; VEGF-A, vascular endothelial growth factor-A.

was overexpressed, VEGF-A expression was upregulated significantly at the mRNA (Fig. 2A and B) and protein (Fig. 2C and D) levels $(\mathrm{P}<0.05)$. Conversely, subsequent to silencing FAP expression in U2-OS cells, VEGF-A expression was significantly downregulated at the mRNA (Fig. 2E and F) and protein (Fig. 2G and H) levels. In addition, when FAP in MG63 cells was overexpressed, an increase in the secreted VEGF-A in supernatant was also identified using ELISA (Fig. 2I). When FAP in MG63 cells was silenced, a decline in the VEGF-A in supernatant was also identified with ELISA (Fig. 2J). This suggested that FAP could promote VEGF-A expression in osteosarcoma cells, implying its pro-angiogenic properties.

CM from osteosarcoma cells with alterations in FAP expression affects the angiogenic properties of HUVEC cells. To further confirm the effect of FAP on angiogenesis, the changes in the angiogenic properties of HUVEC cells were directly assessed. As demonstrated in Fig. 3, subsequent to treatment with CM from MG63 cells with over-expressed FAP, compared with the control group, the phosphorylation of AKT and ERK of HUVEC cells were activated (Fig. 3A). The proliferation rate of the HUVECs increased from $0.89 \pm 0.13$ to $1.32 \pm 0.08$, representing a significant difference (Fig. 3B; $\mathrm{P}<0.05)$. Conversely, subsequent to treatment with $\mathrm{CM}$ from $\mathrm{U} 2-\mathrm{OS}$ cells with silenced FAP for $24 \mathrm{~h}$, the proliferation rate of HUVEC cells declined from $0.92 \pm 0.07$ to $0.44 \pm 0.07$, a significant difference (Fig. $3 \mathrm{C}$; $\mathrm{P}<0.05$ ). This further demonstrated that FAP expression promoted angiogenesis.

Phosphoinositide 3-kinase (PI3K)/AKT and ERK signaling pathways are required for the VEGF-A upregulation induced by FAP. To further investigate the potential molecular mechanisms of FAP in angiogenesis, AKT and ERK were selected 

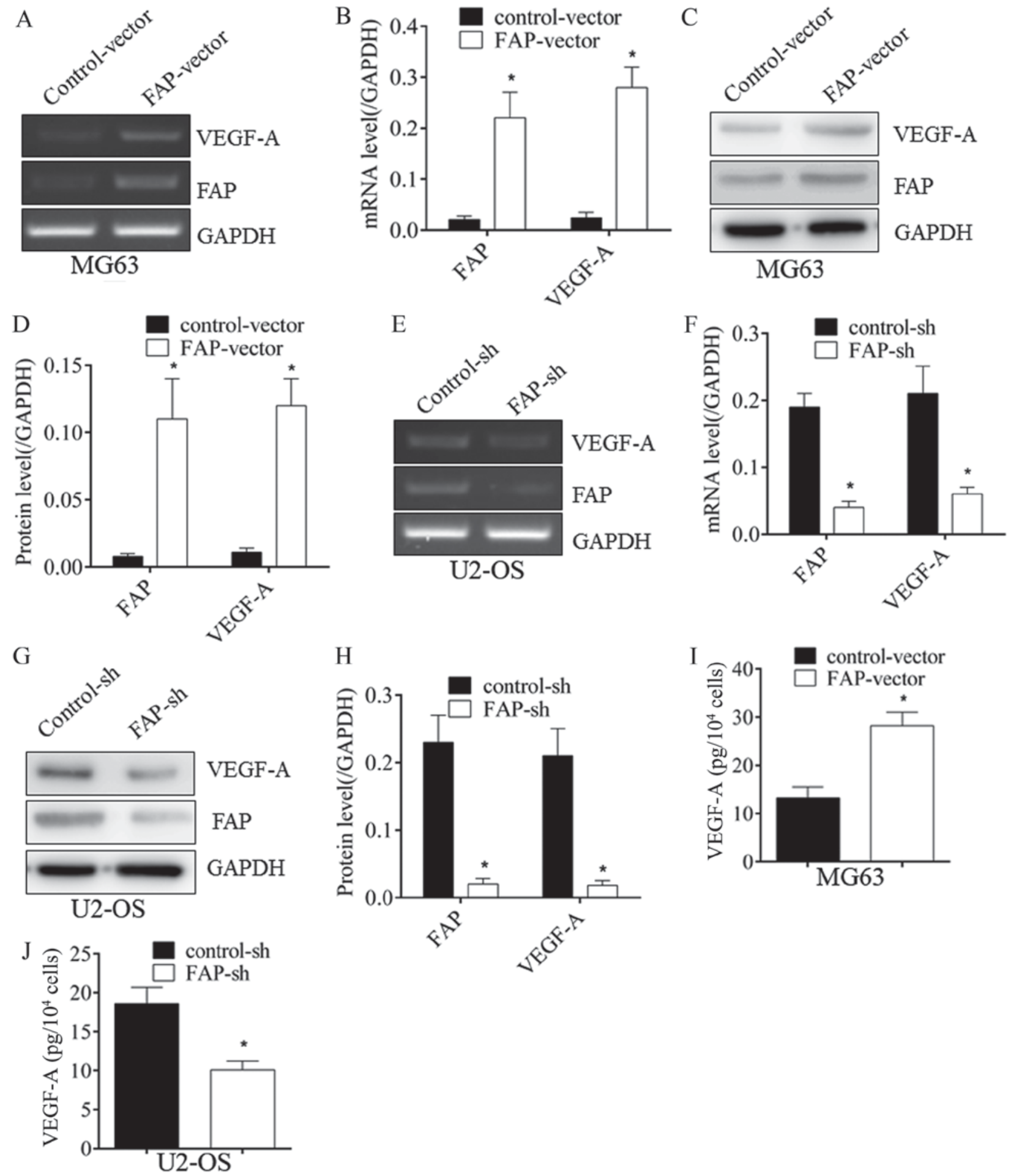

Figure 2. FAP expression promotes VEGF-A expression in osteosarcoma cells. (A) Subsequent to the overexpression of FAP in MG63 cells, VEGF-A mRNA expression was upregulated, as determined with RT-PCR, which was (B) quantified by densitometry. (C) In addition, VEGF-A protein expression was upregulated, which was also (D) quantified. (E) Following the silencing of FAP expression in U2-OS cells, the expression of VEGF-A was inhibited at the mRNA level, as determined with RT-PCR, which was (F) quantified, and at (G) the protein level, which was (H) quantified. (I) The increase in VEGF-A subsequent to FAP overexpression and $(\mathrm{J})$ the decrease in VEGF-A subsequent to FAP silencing were also confirmed by ELISA. "P<0.05 vs. control. FAP, fibroblast activation protein; VEGF-A, vascular endothelial growth factor-A; RT-PCR, reverse transcription-polymerase chain reaction; sh, short hairpin RNA.

as candidate targets. A western blot demonstrated that MG63 cells over-expressing FAP exhibited the activation (phosphorylation) of AKT and ERK (Fig. 4A), whereas in U2-OS cells with silenced FAP expression, the phosphorylation of AKT and ERK were inhibited (Fig. 4B), implying that AKT and ERK may be downstream targets of FAP. Subsequent to treatment with an ERK inhibitor (U0126) or AKT inhibitor (LY294002), the upregulation of VEGF-A expression induced by FAP overexpression was abrogated (Fig. $4 \mathrm{C}$ and D). These results collectively suggest that FAP regulated VEGF-A expression in osteosarcoma cells via the PI3K/AKT and ERK signaling pathways. 
A
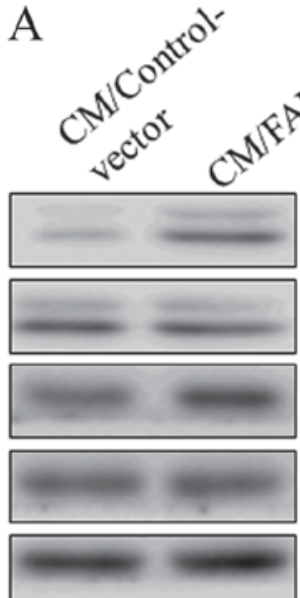

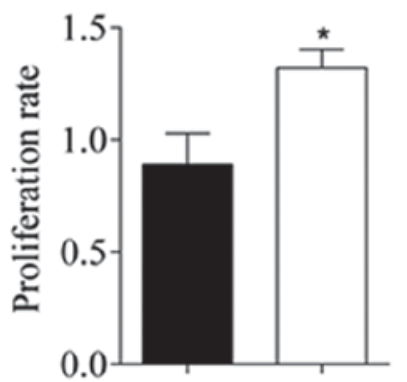

B

AKT

ERK

GAPDH
p-AKT

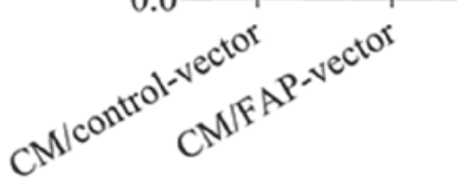

C
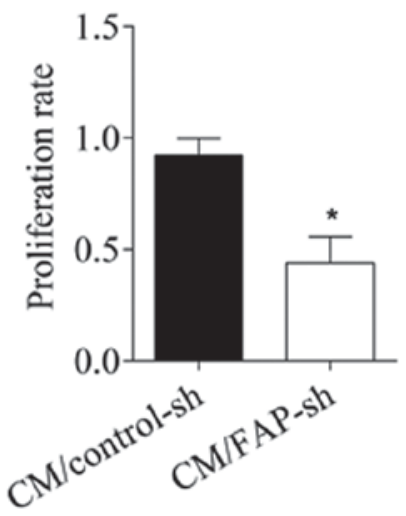

Figure 3. CM from osteosarcoma cells with altered FAP expression can affect the angiogenic properties of HUVECs. (A) Subsequent to treatment using CM from MG63 cells with overexpressed FAP, the phosphorylation of AKT and ERK in HUVECs was activated. (B) HUVEC proliferation rate was significantly increased. (C) Subsequent to treatment using CM from U2-OS cells with silenced FAP, the proliferation rate of HUVECs significantly declined. "P $<0.05$ vs. control. CM, conditioned medium; FAP, fibroblast activation protein; HUVEC, human umbilical vein endothelial cells; ERK, extracellular signal-regulated kinase; p-, phosphorylated-; sh, short hairpin RNA.

A

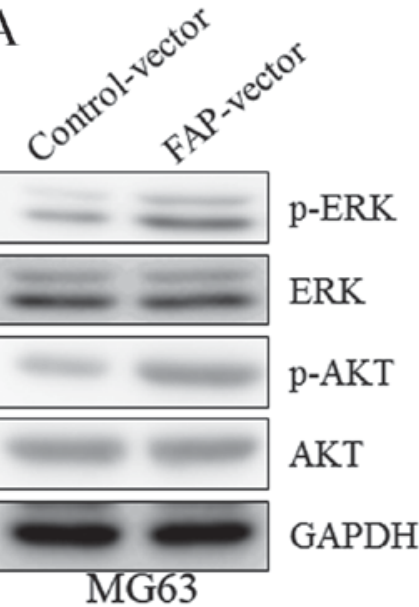

$\mathrm{C}$

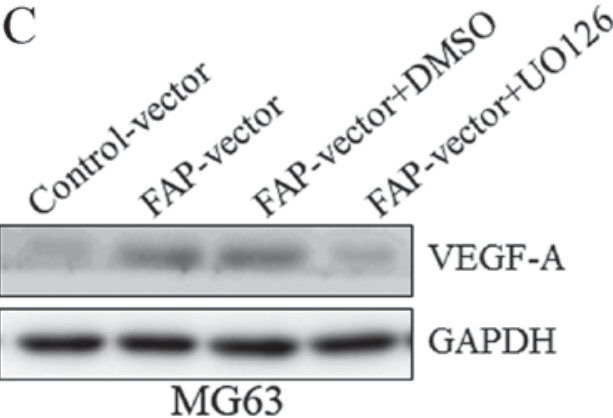

B

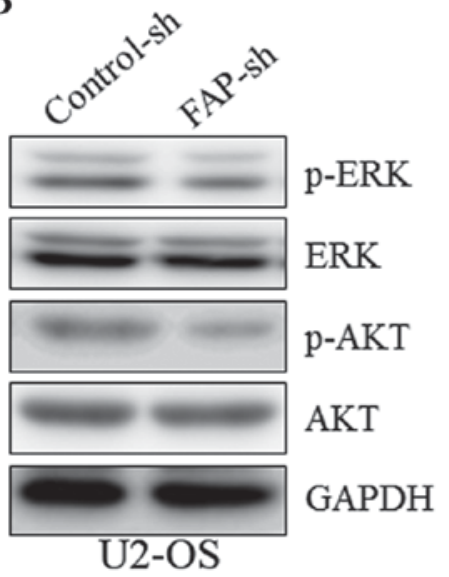

D

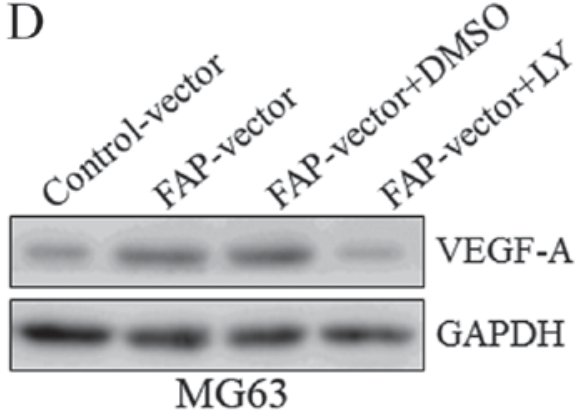

Figure 4. AKT and ERK signaling pathways are required for the upregulation of VEGF-A induced by FAP expression. (A) Subsequent to the overexpression of FAP in MG63 cells, the phosphorylation of AKT and ERK was activated. (B) Subsequent to silencing FAP expression in U2-OS cells, the phosphorylation of AKT and ERK were inhibited. Treatment with (C) the ERK inhibitor U0126 or (D) the AKT inhibitor LY294002 inhibited the upregulation of VEGF-A induced by the overexpression of FAP in MG63 cells. ERK, extracellular signal-regulated kinase; VEGF-A, vascular endothelial growth factor-A; FAP, fibroblast activation protein; p-, phosphorylated; DMSO, dimethyl sulfoxide (control).

\section{Discussion}

Angiogenesis is a complicated process by which new vessels form from existing vasculature. This process is controlled by the balance between proangiogenic factors and antiangiogenic factors (7). VEGF-A is a crucial proangiogenic factor that is implicated in the carcinogenesis of multiple types of tumor (15). It stimulates signaling pathways in endothelial cells through binding to vessel endothelial growth factor receptor 2 (VEGFR-2) to enhance the proliferation, migration 
and tubule formation abilities of the cells, thus promoting angiogenesis (16-18).

FAP, initially emerging as a specific marker of reactive fibroblasts in tumors, is a $170-\mathrm{kDa}$ homodimeric glycoprotein composed of two $97-\mathrm{kDa}$ subunits (19). It belongs to the serine protease family and has dipeptidyl peptidase and endopeptidase activity, allowing the degradation of gelatin and type I collagen (11). The roles of FAP in tumor progression have been widely studied. Jia et al (20) identified that overexpressing FAP significantly promoted the growth and motility of MDA-MB-213 human breast cancer cells in vitro. Shi et al (21) reported that FAP was highly expressed in carcinoma cells, and was associated with desmoplasia and a worse prognosis in pancreatic ductal adenocarcinoma. In the present study, FAP and VEGF-A expression levels were determined in MG63, U2-OS and HOS cells. It was identified that FAP expression was consistent with VEGF-A expression in the different osteosarcoma cell lines. MG63 cells exhibited the lowest expression of FAP and VEGF-A; U2-OS cells exhibited the highest expression. This may imply that FAP expression is correlated with VEGF-A expression. According to the FAP expression level, MG63 and U2-OS cells were selected for further research. Following the silencing of FAP expression in U2-OS cells, VEGF-A expression was significantly downregulated; likewise, over-expressing FAP significantly upregulated the expression of VEGF-A in MG63 cells. To the best of our knowledge, this confirmed the effect of FAP on promoting the expression of pro-angiogenic factors for the first time, further implying its pro-angiogenic role in osteosarcoma.

Vessel endothelial cells line the inner walls of blood vessels and serve a critical role in angiogenesis (22). Under the control of angiogenic factors derived from multiple types of cell, including pericytes and tumor cells, vessel endothelial cells began to proliferate and migrate, resulting in angiogenesis $(7,23)$. In the present study, it was identified that VEGF-A in the supernatant was altered with changes in the FAP expression of osteosarcoma cells. Subsequent to culture in CM from MG63 cells overexpressing FAP, the AKT and ERK signal pathways in HUVEC cells were activated, and the proliferation rate was increased. Furthermore, CM from U2-OS cells with silenced FAP expression inhibited the proliferation rate of HUVEC cells. This demonstrated that the expression of FAP in osteosarcoma cells could, at least in part, promote angiogenesis through regulating the expression of VEGF-A. Whether other angiogenic factors may also be induced by FAP requires further investigation.

The phosphorylation of AKT and ERK has been reported in multiple cancers. They are members of critical signal pathways for maintaining the malignancy of tumor cells, and affect a range of biological behaviors in tumor cells, including proliferation, migration, differentiation, drug resistance, apoptosis and phenotype maintenance (24-26). Liu et al (27) reported that microRNA-21 induced angiogenesis through the activation of AKT and ERK in DU145 human prostate cancer cells. Wang et al (28) identified that the downregulation of FAP suppressed cell proliferation and metastasis through the PI3K/AKT and ERK signaling pathways in oral squamous cell carcinoma. In the present study, it was identified that the phosphorylation of AKT and ERK were activated by FAP. The Akt inhibitor, LY294002, and the ERK inhibitor, U0126, each abrogated the upregulation of VEGF-A induced by FAP. This is consistent with previous studies $(27,28)$. However, as there is complexity in the crosstalk between different signaling pathways, although the activation of AKT and ERK were confirmed in the present study, the detailed mechanism of regulation has yet to be fully elucidated.

In conclusion, FAP and VEGF-A expression levels corresponded with each other in osteosarcoma cells. The overexpression of FAP in osteosarcoma cells activated the PI3K/AKT and ERK signaling pathways, which may promote angiogenesis. This data may enrich the understanding of the tumor-promoting role of FAP, and supplies evidence to support the identification of novel therapy targets against osteosarcoma.

\section{Acknowledgements}

Not applicable.

\section{Funding}

No funding was received.

\section{Availability of data and materials}

All data generated or analyzed during this study are included in this published article

\section{Authors' contributions}

CZ designed the present study and wrote the paper; MW performed the cell experiments and XL performed the cell experiments and data analysis.

\section{Ethics approval and consent to participate}

Not applicable.

\section{Consent for publication}

Not applicable.

\section{Competing interests}

The authors declare that they have no competing interests.

\section{References}

1. Savage SA, Mirabello L, Wang Z, Gastier-Foster JM, Gorlick R, Khanna C, Flanagan AM, Tirabosco R, Andrulis IL, Wunder JS, et al: Genome-wide association study identifies two susceptibility loci for osteosarcoma. Nat Genet 45: 799-803, 2013.

2. Bonuccelli G, Avnet S, Grisendi G, Salerno M, Granchi D, Dominici M, Kusuzaki K and Baldini N: Role of mesenchymal stem cells in osteosarcoma and metabolic reprogramming of tumor cells. Oncotarget 5: 7575-7588, 2014.

3. Lu J, Song G, Tang Q, Zou C, Han F, Zhao Z, Yong B, Yin J, $\mathrm{Xu} \mathrm{H}, \mathrm{Xie} \mathrm{X}$, et al: IRX1 hypomethylation promotes osteosarcoma metastasis via induction of CXCL14/NF- $\mathrm{BB}$ signaling. J Clin Invest 125: 1839-1856, 2015.

4. Garcia A and Singh H: Bevacizumab and ovarian cancer. Ther Adv Med Oncol 5: 133-141, 2013.

5. Bergers $\mathrm{G}$ and Benjamin LE: Tumorigenesis and the angiogenic switch. Nat Rev Cancer 3: 401-410, 2003. 
6. Rusckowski M, Wang Y, Blankenberg FG, Levashova Z, Backer MV and Backer JM: Targeted scVEGF/(177)Lu radiopharmaceutical inhibits growth of metastases and can be effectively combined with chemotherapy. EJNMMI Res 6: 4, 2016.

7. Hoeben A, Landuyt B, Highley MS, Wildiers H, Van Oosterom AT and De Bruijn EA: Vascular endothelial growth factor and angiogenesis. Pharmacol Rev 56: 549-580, 2004.

8. Zi FM, He JS, Li Y, Wu C, Wu WJ, Yang Y, Wang LJ, He DH, Yang L, Zhao Y, et al: Fibroblast activation protein protects bortezomib-induced apoptosis in multiple myeloma cells through $\beta$-catenin signaling pathway. Cancer Biol Ther 15: 1413-1422, 2014.

9. Wikberg ML, Edin S, Lundberg IV, Van Guelpen B, Dahlin AM, Rutegård J, Stenling R, Oberg A and Palmqvist R: High intratumoral expression of fibroblast activation protein (FAP) in colon cancer is associated with poorer patient prognosis. Tumor Biol 34: 1013-1020, 2013.

10. Hua X, Yu L, Huang X, Liao Z and Xian Q: Expression and role of fibroblast activation protein-alpha in microinvasive breast carcinoma. Diagn Pathol 6: 111, 2011.

11. Liu R, Li H, Liu L, Yu J and Ren X: Fibroblast activation protein Cancer Biol Ther 13: 123-129, 2014.

12. Lai D, Ma L and Wang F: Fibroblast activation protein regulates tumor-associated fibroblasts and epithelial ovarian cancer cells. Int J Oncol 41: 541-550, 2012.

13. Mentlein R, Hattermann K, Hemion C, Jungbluth AA and Held-Feindt J: Expression and role of the cell surface protease seprase/fibroblast activation protein- $\alpha$ (FAP- $\alpha)$ in astroglial tumors. Biol Chem 392: 199-207, 2011.

14. Yuan D, Liu B, Liu K, Zhu G, Dai Z and Xie Y: Overexpression of fibroblast activation protein and its clinical implications in patients with osteosarcoma. J Surg Oncol 108: $157-162,2013$

15. Zhao D, Pan C, Sun J, Gilbert C, Drews-Elger K, Azzam DJ, Picon-Ruiz M, Kim M, Ullmer W, El-Ashry D, et al: VEGF drives cancer-initiating stem cells through VEGFR-2/Stat3 signaling to upregulate Myc and Sox2. Oncogene 34: 3107-3119, 2015.

16. Ishigami SI, Arii S, Furutani M, Niwano M, Harada T, Mizumoto M, Mori A, Onodera $\mathrm{H}$ and Imamura M: Predictive value of vascular endothelial growth factor (VEGF) in metastasis and prognosis of human colorectal cancer. Br J Cancer 78 : $1379-1384,1998$

17. Neufeld G, Cohen T, Gengrinovitch S and Poltorak Z: Vascular endothelial growth factor (VEGF) and its receptors. FASEB J 13: 9-22, 1999
18. Cross MJ, Dixelius J, Matsumoto T and Claesson-Welsh L: VEGF-receptor signal transduction. Trends Biochem Sci 28: 488-494, 2003.

19. Kelly T, Huang Y, Simms AE and Mazur A: Fibrobl Activation Protein- $\alpha$ : A key modulator of the microenvironment in multiple pathologies. Int Rev Cell Mol Biol 297: 83-116, 2012.

20. Jia J, Martin TA, Ye L and Jiang WG: FAP- $\alpha$ (Fibroblast activation protein- $\alpha$ ) is involved in the control of human breast cancer cell line growth and motility via the FAK pathway. BMC Cell Biol 15: 16, 2014.

21. Shi M, Yu DH, Chen Y, Zhao CY, Zhang J, Liu QH, Ni CR and Zhu MH: Expression of fibroblast activation protein in human pancreatic adenocarcinoma and its clinicopathological significance. World J Gastroenterol 18: 840-846, 2012.

22. Adeoye OO, Bouthors V, Hubbell MC, Williams JM and Pearce WJ: VEGF receptors mediate hypoxic remodeling of adult ovine carotid arteries. J Appl Physiol (1985) 117: 777-787, 2014

23. Wu Y, Huang A, Li T, Su X, Ding H, Li H, Qin X, Hou L, Zhao Q, Ge X, et al: MiR-152 reduces human umbilical vein endothelial cell proliferation and migration by targeting ADAM17. FEBS Lett 588: 2063-2069, 2014.

24. Steelman LS, Chappell WH, Abrams SL, Kempf RC, Long J, Laidler P, Mijatovic S, Maksimovic-Ivanic D, Stivala F, Mazzarino MC, et al: Roles of the Raf/MEK/ERK and $\mathrm{PI} 3 \mathrm{~K} / \mathrm{PTEN} / \mathrm{Akt} / \mathrm{mTOR}$ pathways in controlling growth and sensitivity to therapy-implications for cancer and aging. Aging (Albany NY) 3: 192-222, 2011.

25. Sasore $\mathrm{T}$ and Kennedy B: Deciphering combinations of $\mathrm{PI} 3 \mathrm{~K} / \mathrm{AKT} / \mathrm{mTOR}$ pathway drugs augmenting anti-angiogenic efficacy in vivo. PLoS One 9: e105280, 2014.

26. Khan KH, Yap TA, Yan L and Cunningham D: Targeting the PI3K-AKT-mTOR signaling network in cancer. Chin J Cancer 32: 253-265, 2013

27. Liu LZ, Li C, Chen Q, Jing Y, Carpenter R, Jiang Y, Kung HF, Lai L and Jiang BH: miR-21 induced angiogenesis through AKT and ERK Activation and HIF-1 $\alpha$ expression. PLoS One 6: e19139, 2011.

28. Wang H, Wu Q, Liu Z, Luo X, Fan Y, Liu Y, Zhang Y, Hua S, Fu Q, Zhao M, et al: Downregulation of FAP suppresses cell proliferation and metastasis through PTEN/PI3K/AKT and Ras-ERK signaling in oral squamous cell carcinoma. Cell Death Dis 5: e1155, 2014. 